\title{
Learning to capture the functions of genetic regulatory networks using graph motifs
}

\author{
V.K. Murthy and E.V. Krishnamurthy* \\ Australian National University, \\ Canberra, ACT 0200, Australia \\ E-mail: edayathuk@gmail.com \\ E-mail: Evk.Krishnamurthy@anu.edu.au \\ *Corresponding author
}

\begin{abstract}
Network motifs have been suggested as a means to understand Systems biological functions. Based on several arguments from computer science, logic, mathematics, chemistry and non-linear dynamics, we explain the difficulties encountered in capturing the evolving dynamical functions carried out by the genetic regulatory networks (GRN), based on the assembly of motifs.
\end{abstract}

Keywords: adjacency matrices; assortative networks; complex network; CN; disassortative networks; eigenvalues; graphs; Lyapunov exponents; motifs; positive entropy; system biology.

Reference to this paper should be made as follows: Murthy, V.K. and Krishnamurthy, E.V. (2012) 'Learning to capture the functions of genetic regulatory networks using graph motifs', Int. J. Advanced Intelligence Paradigms, Vol. 4, No. 2, pp.185-197.

Biographical notes: V.K. Murthy is an Academic Visitor at the Australian National University, Canberra. He has published several papers and books about computer science, information systems, and agent-based software and simulation.

E.V. Krishnamurthy is currently with the Australian National University, Canberra. He has contributed widely in several areas in computer science, chemical information systems and complex systems.

\section{Introduction}

Various attempts are currently made using the machine learning approach to understand the structure and function of complex networks $(\mathrm{CNs})$ in systems biology, ecosystems, food-web and chemical structures These include attempts to use machine learning in extracting motifs - small fragment subgraphs of the relevant $\mathrm{CN}$ using graph kernels and related approaches - such as random walk, convolution kernels (Kashima et al., 2004; Rupp and Schneider, 2010; Rupp, 2011). These attempts are reminiscent of the attempts over a number of years to name chemical compounds based on such subgraph concepts, as for example the well-known Wiswesser line notation (WLN) and its variants, (Krishnamurthy et al., 1974; Krishnamurthy and Lynch, 1981) Similar approaches have 
been made for patent analysis of chemical compounds, based on the powerful relaxation screening (Kitchen and Krishnamurthy, 1982). These involve very complex substructure search and have been only partially successful in the sense of narrowing down the search from application point of view. Also many attempts have been made to obtain a chemical name from which substructures can be inferred so that they can be useful for design and analysis of chemical compounds with applications to antibiotics, cosmetics, food, fertilisers and medicine. In all the above cases, we essentially require static shape matching rather than inferring the dynamical functions they carry out. To infer these higher-level functions, we require both in vivo and in vitro experiments, since structure and function need not always be related. Although some substructures may be sufficient to infer certain functions, this may not always be necessary, and further there are many cases in which different substructures can exhibit same phenomena, as for example, especially in toxins and venoms. Further, most of the results obtained on these aspects that are based on machine learning using statistical correlation are only statistically acceptable within some confidence limits.

\section{The motif approach}

The graph motif approach in genetic regulatory networks (GRNs) was pioneered by Alon et al. (2007a, 2007b). In a very lucid book, Alon et al. (2007a) has examined transcription, transduction and communication networks in systems biology and has found that the following primitives are universally present: feed-forward loops (FFL) both coherent and incoherent, autoregulation (single loop), multi-output FFL, Bifan.

The graph motif approach may resemble a structured programme primitive. However, it must be remembered that structured programme primitives are functionally complete, based on two-valued logical functions or number theoretic computable functions, while in the case of graph motifs we deal with non-linear real functions that are stochastic (Scott, 2007). In this sense, the motifs provide only a rudimentary syntax for the system biology.

Further, it is to be noted that the definition of functions in systems biology is entirely different from computable or mathematical functions (Manna, 1974). Three kinds of interrelated functions occur in systems biology:

1 the biochemical function referring to the chemical activity, binding and catalytic property and conformational changes

2 the cellular function that is context dependent with respect to a tissue or an organ

3 the phenotypic function that determines the behavioural and physiological properties of an organism in its environment.

In language theory, the above three functional aspects are analogous to the syntax that deals with the structure and grammar of a sentence (the chemical structure, bonds and reaction), the semantics that assigns a meaning to a sentence (its role in cellular action), and thirdly the pragmatics of a language which deals with the usability of the language in successfully meeting its goals (survival of an organism). All these functions are non-unique and context dependent. That is, similar functions can result in different outcomes and different functions can result in similar outcomes due to their many to one, and one too many mapping properties. This is called multiple realisability and leads to the failure of reductionism. 
In mathematical logic these functions correspond to higher-order logical functions that deal with the non-denumerable properties of functions of another function of yet another function, and hence turn out to be non-computable. In addition, proteins are multi domain structures, undergoing extensive conformational changes and rapidly evolve their folds through order-disorder transitions making the complexity of folding problems in proteins computationally very complex.

\section{Motif approach: problems encountered}

Ingram et al. (2006) and Knabe et al. (2007) have pointed out the difficulty in motif approach to determine the evolved function from subgraph structure. They show that that the dynamics of each motif can exhibit a wide range of dynamical responses and with the parameter variation, they can indeed exhibit even opposite behaviour. We elaborate further on this aspect based on other arguments.

\subsection{Computability viewpoint}

As mentioned earlier, the assemblage of motifs look somewhat analogous to the modular software construction. In reality, the motifs are not independent blocks and have positive and negative feedback processes leading to non-linear dynamics that generate positive metric entropy and positive Lyapunov exponents. Accordingly, the system is no longer separable into individual algorithmic modules and emerges into a qualitatively new system that is either stochastic or chaotic (Mosekilde and Mosekildae, 1991).

\subsection{Motifs behaviour in system biology}

In systems biology the components are chemical concentration-based and the activation of any component is based on the threshold of chemical concentration, as well as, its rate of change. In fact the activation function that results from two other concentrations is a weighted sum. This activation function when subjected to a threshold can decide whether the ultimate outcome is an activation or inhibition. In fact a given motif can compute various logical functions or other functions depending upon the weights and the threshold. To illustrate this we consider examples of simple one layer and two layer neural networks.

It is well-known that a neural network consists of an input set of variables usually real numbers. These inputs are then multiplied by weighting functions and added. The resulting sum is then compared against a threshold function $\mathrm{t}$; if the sum is greater than or equal to $t$ the output is one; otherwise zero. A single layer neural network consists of a single set of inputs, weighting functions and summing device and a threshold function as described above. If these arrangements are repeated at several level it is called a multilayer neural network.

For example, if a threshold neural logic gate receives inputs $\mathrm{x} 1$ and $\mathrm{x} 2$ and if these are weighted by multipliers a1 and a2 respectively and added to form $y=a 1 . x 1+a 2 . x 2$, then the functions computed by this logic gate for the following cases, using the threshold function $\mathrm{t}(\mathrm{y})$ will be analysed below. We assume that by $0 \leq \mathrm{x} 1 \leq 1$, and $0 \leq \mathrm{x} 2 \leq 1$. The output region of these networks depend on the choice of the parameters (weights) a1 and 
a2. These weights along with the choice of the threshold play a role in determining the activation function.

Case $1 \mathrm{x} 1$ and $\mathrm{x} 2$ are either 0 or 1 ; $\mathrm{a} 1=\mathrm{a} 2=0.7$, If $\mathrm{t}(\mathrm{y}) \geq 0.8$, the output is 1 ; otherwise the output is 0 . This is logical AND.

Case $2 \mathrm{x} 1$ and $\mathrm{x} 2$ are either 0 or 1 ; $\mathrm{a} 1=\mathrm{a} 2=0.1$, If $\mathrm{t}(\mathrm{y}) \geq 0.1$, the output is 1 ; otherwise the output is 0 . This is logical OR.

Case $3 \mathrm{x} 1$ and $\mathrm{x} 2$ are either 0 or 1 ; $\mathrm{a} 1=\mathrm{a} 2=-0.5$. If $\mathrm{t}(\mathrm{y}) \geq-0.1$ the output is 1 ; otherwise the output is 0 . This is logical NOR.

Case $4 \mathrm{x} 1$ and $\mathrm{x} 2$ are either 0 or $1 ; \mathrm{a} 1=0.5, \mathrm{a} 2=-0.5$. If $\mathrm{t}(\mathrm{y}) \geq-0.8$ the output is 1 ; otherwise the output is 0 . This is a logical NAND.

Case $5 \mathrm{x} 1$ and $\mathrm{x} 2$ are either 0 or $1 ; \mathrm{a} 1=0.5, \mathrm{a} 2=-0.5$. If $\mathrm{t}(\mathrm{y}) \geq 0.1$ the output is 1 ; otherwise the output is 0 . This is a logical function ( $\mathrm{x} 1$ and not $\mathrm{x} 2$ ).

Case 6 Restrict to a single input by setting: $\mathrm{x} 1=0$.

Then with $\mathrm{a} 1=0, \mathrm{x} 2$ is 0 or $1 ; \mathrm{a} 2=-0.5$, and the condition if $\mathrm{t}(\mathrm{y}) \geq-0.1$, then output is 1 ; otherwise 0 . Then the outputs when $\mathrm{x} 2=0$ or 1 will give a logical NOT.

Consider now a two layer neural network consisting of the following inputs and weights at the two-levels.

Inputs: (x1, x2), weights a1, a2, b1, b2, c1, c2 and summing over takes place thus:

$$
\mathrm{y} 1=\mathrm{a} 1 \cdot \mathrm{x} 1+\mathrm{b} 2 \cdot \mathrm{x} 2 ; \mathrm{y} 2=\mathrm{b} 1 \cdot \mathrm{x} 1+\mathrm{a} 2 \cdot \mathrm{x} 2 ; \text { and } \mathrm{z}=\mathrm{c} 1 \cdot \mathrm{y} 1+\mathrm{c} 2 \cdot \mathrm{y} 2 \text {. }
$$

Let $\mathrm{x} 1, \mathrm{x} 2$ can be either 0 or 1 for the following values of a1, a2.b1, b2, c1 and c2, and the following thresholds $t(y 1), t(y 2), t(z)$.

$$
\mathrm{a} 1=\mathrm{a} 2=\mathrm{b} 1=\mathrm{b} 2=1 ; \mathrm{c} 1=0.6 \text { and } \mathrm{c} 2=-0.2
$$

If $\mathrm{t}(\mathrm{y} 1) \geq 0.4$ the output is 1 ; otherwise 0 . If $\mathrm{t}(\mathrm{y} 2) \geq 1.2$, its output is 1 ; otherwise 0 .

Also; $\mathrm{t}(\mathrm{z}) \geq 0.5$ it outputs 1 ; otherwise 0 .

The function computed is exclusive OR function. This is because:

Input $(\mathrm{x} 1, \mathrm{x} 2)=(0,0)$ : then $\mathrm{y} 1=0, \mathrm{y} 2=0$ and so output $\mathrm{t}(\mathrm{y} 1)=0$,

and output of $\mathrm{t}(\mathrm{y} 2)=0$.

Then $\mathrm{z}=0$ and its output is 0 .

$\operatorname{Input}(\mathrm{x} 1, \mathrm{x} 2)=(0,1)$ : then $\mathrm{y} 1=1, \mathrm{y} 2=1$ and so output of $\mathrm{t}(\mathrm{y} 1)=1$,

and output of $\mathrm{t}(\mathrm{y} 2)=0$, since $\mathrm{t}(\mathrm{y} 2)$ is 1.2 .

Then $\mathrm{z}=0.6-0.0=0.6 \geq 0.5$; so its output is 1 .

$\operatorname{Input}(\mathrm{x} 1, \mathrm{x} 2)=(1,0)$ : then $\mathrm{y} 1=1, \mathrm{y} 2=1$ and so output of $\mathrm{t}(\mathrm{y} 1)=1$,

output of $\mathrm{t}(\mathrm{y} 2)=0$ since $\mathrm{t}(\mathrm{y} 2)$ is 1.2 .

Then $\mathrm{z}=0.6-0=0.6 \geq 0.5$. Hence output is 1 .

$\operatorname{Input}(\mathrm{x} 1, \mathrm{x} 2)=\operatorname{Input}(1,1)$ : then $\mathrm{y} 1=2, \mathrm{y} 2=2$ and so output of $\mathrm{t}(\mathrm{y} 1)=1, \mathrm{t}(\mathrm{y} 2)=1$, and so $\mathrm{z}=0.6-0.2=0.4<0.5$ and output is 0 . 
Thus an identically looking motif can generate various functions in various different ways, and the many layer networks can produce different outputs depending upon the chemical concentration levels and threshold functions. If the inputs and outputs are stochastic in nature, the functions computed are no longer easily determinable. Further any differing performance even in a single motif among a set of identical motifs in a network will propagate through the network, as stated in Moran's theorem (next subsection).

\subsection{Moran's theorem}

In a biological system that is stochastic (Jiang et al., 2006) any slightly mutated individual motif (or block) with a differing performance will tend to propagate though the system and will dominate with its own fixation probability. This is Moran's theorem (Nowak, 2006). Moran process is the simplest possible stochastic model to study selection in a finite population. Hence, any mutant that arises in a population with a higher fitness will eventually take over the population with a high fixation probability. The selective force is so strong that the function of the system will be different from what one wanted it to be. As a result, identical motifs that may have varying stochastic inputs, when composed, can generate chemical and biological functions that may not be identical.

\subsection{Graph structures}

The realisation of a total graph based on composition of subgraphs (or other strongly connected components) is operationally easy. However, the spectral radius (eigenvalue range) of the composite graph cannot be easily determined, unless the graph is organised as individual block graphs with minimal (or weak) connections between blocks, since only block matrices can have eigenvalues of the individual blocks. If there are many off diagonal elements that correspond to the new links (however, weak) among the subgraphs, the resulting graph will have widely differing eigenvalues, and hence the resulting dynamical system behaviour is in general, will be unpredictable, except for deterministic known links. Thus, the principle of reductionism does not hold in matrix eigenvalue theory, as the spectral transitions are unpredictable, in general (Bornholdt and Schuster, 2003; Palla and Vattay, 2006).

For example, if the motif graphs are densely connected, the eigenvalues can differ widely, and this will affect their Lyapunov exponents, while functioning in a dynamic environment. This can result in totally different dynamical functions.

\subsection{Proving dynamic functionality}

In software engineering, all software will contain structured programming primitives, such as, assignment, decision, if, while loop and for loop. A statistical analysis will give these as primitives - from which no one can guess what the computed function is or whether the programme will ever halt. These structured primitives have been designed for ease in programming for freedom from error. Using these primitives as the basis, programme correctness is provable (Manna, 1974). On the other hand, in the case of GRN, the assembly of motifs or a modular approach will not enable us to mathematically 
prove that the assembled graph has the same dynamical functionality as the original network. Simulation might provide a solution to a particular case - but not provide a proof.

\subsection{Reductionism and emergence}

As observed earlier biological and many other complex systems are not amenable to decomposition into subsystems and reassembling into a total system due to their intrinsic emergent properties. Even very simple lattice models, such as Bak's sand pile and Ising model exhibit self-organisation that is not predictable (Murthy and Krishnamurthy, 2009). Therefore, it is not feasible according to the reductionist argument that system biology can be reduced to a few elementary components without considering the global environment in which it is embedded.

\subsection{Non-linear dynamics}

In the physics of non-linear dynamical systems, it is well known that even deterministic systems can exhibit unpredictability due to chaos and positive metric entropy. The properties of a $\mathrm{CN}$ are governed by three important parameters: metric entropy, Lyapunov exponents, and fractal dimension. There is a close relationship between positive Lyapunov exponents (or equivalently the eigenvalues of the interaction matrix) and fractal dimensions. Lyapunov exponents measure how the trajectories move apart and they are measured as bits per second. If the Lyapunov exponent is positive the rate at which information is generated increases exponentially and we need to measure increasing amount of information. If the Lyapunov exponent is negative, we need to measure decreasing amount of information over time as we are moving towards a stable state.

If $\mathrm{h}(1), \mathrm{h}(2), \ldots, \mathrm{h}(\mathrm{j})$ are Lyapunov exponents in ascending order that are greater than zero, and, $h(j+1), \ldots h(k)$ are less than zero in descending order, then we have $\mathrm{j}$ stretching and $(\mathrm{k}-\mathrm{j})$ contracting directions in the $\mathrm{k}$ dimensional trajectories, then the quantity, Lyapunov dimension, (Murthy and Krishnamurthy,2009)

$$
d(L)=j+1 /|h(j+1)| \sum_{i=1}^{j} h(i) .
$$

It is conjectured that $\mathrm{d}(\mathrm{L})=\mathrm{d}(\mathrm{b})$ the box-counting dimension.

Another dimension that is used is the topological dimension. Here we add up the positive and negative Lyapunov exponents until the sum is just greater than or zero. The largest number $\mathrm{j}$ of exponents $\mathrm{h}(\mathrm{j})$ added to keep the sum non-negative is the topological dimension $\mathrm{D}$. 2009):

The Kaplan-Yorke dimension D (KY) is then defined by (Murthy and Krishnamurthy,

$$
D(K Y)=D+1 /|h(j+1)| \sum_{i=1}^{j} h(i),
$$

If there are no negative Lyapunov exponents the system is unbounded; if all the exponents sum to zero the system is volume conserving and usually structurally unstable, 
unless the system is energy conserving. For non-dissipative conservative system, the Lyapunov exponents occur as a pair of negative and positive exponents of equal values that sum to zero. The system is then time reversible.

In dissipative systems the sum of Lyapunov exponents is negative with at least one negative Lyapunov exponent; then the state space contracts and collapses onto an attractor.

The fractal dimensions $\mathrm{d}(\mathrm{L})$ and $\mathrm{D}(\mathrm{KY})$ can change rapidly depending upon $\mathrm{h}(\mathrm{i})$ as a function of the network size, the nature of the connectivity structure and the interaction among the components. The Lyapunov exponents then determine the stability or instability of the system and emergence (Scott, 2007; Murthy and Krishnamurthy, 2009).

In multidomain proteins, each domain has a compact globular structure and is an independent folding unit; further all the different domains can fold simultaneously. From the graph theory point of view, the residues within a domain are more densely connected than with the residues in different domains, and different domains are sparsely connected. In this case, the adjacency matrix of the protein graph nearly assumes a block diagonal form. The dynamical stability of the total system is essentially governed by the stability of each individual domain, and the small positive Lyapunov exponents in each domain are nearly unaffected by its neighbouring domains. This network becomes quickly stable reaching the attractor of the system, namely, its native state. However, computing Lyapunov exponents for folding and unfolding of proteins is extremely challenging due to their large phase space dimensionality and the rate at which information is generated. A similar situation arises, when a composite set of motifs are assembled and the system stability is to be inferred.

An interesting example is the simulation of chaotic dynamics of Abductin, a rubber protein, consisting of the sequence of ten amino acids: Phe-Gly-Gly-Met-Gly-Gly-GlyAsn-Ala-Gly, that has a ten-dimensional phase space (Villani, 2003). This system exhibits emergence and hence cannot be described in terms of its simple vibrational and rotational movements. It reaches a self-organising critical state and is chaotic with six positive and four negative Lyapunov exponents. Villani (2003) has computed the Lyapunov exponents in forward and backward in time. These are respectively:

$(0.4815,0.3959,0.3161,0.2346,0.1392,0.0190,-0.1171,-0.3131$, $-0.6109,-1.2779)$

and

$$
\begin{aligned}
& (0.5513,0.4666,0.3772,0.2907,0.1969,0.0786,-0.0576,-0.2505, \\
& -0.5426,-1.1906) .
\end{aligned}
$$

The first of these nine exponents sum to non-negative values, namely: 0.5452 and 1.1106.

The Kaplan-Yorke fractal dimension for forward and backward in time are:

$$
9+(0.5452 / 1.2779)=9.42
$$

and

$$
9+(0.1 .1106 / 1.1906)=9.93 .
$$

This indicates that in dimension 10 the hyper-volume is contracting and the attractor has a Kaplan-Yorke dimension between 9.42 to 9.93 . 
Shakhnovich (2006) observes that long range interactions result in two-state cooperativity, while short range forces interactions accelerate the folding of proteins. Depending upon their spectral properties (Palla and Vattay, 2006), protein networks, under suitable environmental conditions can exhibit either percolation like transition or stability to local fluctuations.

\subsection{Chemical kinetic conflicts}

In systems biology and chemistry, depending upon the temporal order of cooperative binding, varying chemical reactions can occur due to changes in chemical bonds, chirality and thermodynamics. Ingram et al. (2006) demonstrate such examples from gene regulation and protein production. In fact, they show that the characteristic output of a motif can qualitatively be different, according to the detailed structure they are embedded into. That is there is local to global and global to local influence which supports the argument of emergence.

\section{CNs in nature}

We now recall some fundamental properties of $\mathrm{CNs}$ that occur in Nature and examine the role of motifs and why motifs cannot capture the functions of a $\mathrm{CN}$.

CNs (that include GRN) in nature exhibit the following important properties.

\subsection{Interacting components, interaction with environment, energy source}

A CN consists of a large network of number of interacting components and an environment that is defined by a boundary to discriminate the inside (self) from the outside (non-self) of the $\mathrm{CN}$. The components collectively and cooperatively perform actions, the actions being driven by an energy source. These actions are coordinated to obtain maximal efficiency to seek the optimum, under varying environmental conditions and constraints. Thus it is a non-linear dynamic optimisation problem in a non-stationary environment in which the constraints are not fixed and the evaluation of fitness function is not pre-specified.

\subsection{Network exhibits emergence}

We say that a network $\mathrm{N}$ of interrelated components in $\mathrm{CN}$ exhibit an emergent process $\mathrm{E}$ with emergent properties P if and only if (Murthy and Krishnamurthy, 2009):

1 non-linear dynamics: $\mathrm{E}$ is a global process that instantiates $\mathrm{P}$, and arises from the coupling of N's components and the non-linear dynamics D of their local interactions

2 global to local influence: E and P have a global to local (downward) causation or determinative influence on the dynamics $\mathrm{D}$ of the components of $\mathrm{N}$

3 relational Holism: $\mathrm{E}$ and $\mathrm{P}$ are not exhaustively determined by the intrinsic properties of the components of $\mathrm{N}$, that is they exhibit relational holism. 
Emergence has similarities to the phase transition encountered in physics, where local changes result in a global change in which new properties emerge abruptly. In particular, under emergence, the many degrees of freedom arising due to its component parts collapse into a fewer new ones with a smaller number of globally relevant parameters. That is the properties of emergence cannot be predetermined and the system evolves on a qualitatively new level with respect to its global constraints. In this sense, emergence implies the failure of the reductionist hypothesis, which claims that a total system can be analysed in terms of the intrinsic properties of the constituent parts.

\subsection{Power-law scaling or scale-invariance property in emergence}

It means that the given functional form $\mathrm{F}(\mathrm{x})$ remains unchanged to within a multiplicative factor under the rescaling of the independent variable $\mathrm{x}$. That is, such a property results in the equation:

$$
\mathrm{F}(\mathrm{ax})=\mathrm{bF}(\mathrm{x})
$$

namely invariance under multiplicative changes of scale that results in self-similarity. This property can occur in both space and time for the emergent properties of the CNs.

\subsection{Adaptation including fault-tolerance and resilience to damage}

Adaptation is a condition that reflects flexibility or capacity to change in relation to changing conditions in a viable way. $\mathrm{CN}$ can self-modify their past behaviour and adapt to environmental changes, available resources, as well as, tolerate failures or non-cooperation of some of their components, in achieving a specified goal.

Evolutionary theory claims that organisms tend to pass on from generation to generation those properties that are more likely to help them adapt to their environment (as in a Moran process). The organisms carrying out this selection process most efficiently will survive the longest, and that the organisms will tend to find gaps in the natural world in order to evolve in new ways that are not already exhausted by existing organisms. This means that over a period of time, organisms exhibit emergent properties.

Also a mathematical functional or a recursively computable relationship is not derivable between adaptation, and mutation, selection and their interplay. In evolutionary biology, many random mutations and selections take place over a long period of time to adapt to a new environment resulting in the synthesis of new proteins, or stopping of the synthesis, or modification of existing proteins. These result in newer biological functions, such as: sensitivity to ultraviolet radiation, modification of haemoglobin, production of antifreeze, production of venoms, toxins, and loss of visual apparatus in animals living in darkness or evolution of new organs. It is now well established that all these effects arise due to changing chemistry, through mutation of codons, to adapt to a new environment.

Thus, the set of all possible chemical compounds that can evolve during a long period of time, their physical, structural properties (nature of bonds, their angles, energy functions and reactions) and biological activity properties, due to environmental changes turn out to be non-computable or non-algorithmic. Also, the cause-effect relation among them turns out to be circular, as to whether the mutation was the cause or it is an effect due to other means. Further, most functional properties cannot be predicted in advance, and can only be analysed after they have happened. 
In summary, there are good reasons to believe that the above four properties are interrelated to self-organisation, stigmergy and self-assembly, as well as, to systems exhibiting positive metric entropy, self-similarity and the small world phenomena. It is hard to separate these concerns as they form fuzzy boundaries (Murthy and Krishnamurthy, 2009).

Also, it is well accepted that most CNs in nature are far from being random networks. This does not imply that they are amenable to structural decomposition and reassembly. These CNs widely vary in their interactive connective structure and they have widely differing average degree, characteristic path length, cluster coefficient. Some are scale free, some have small world property (Boccaletti et al., 2006; Newman, 2004; Murthy and Krishnamurthy, 2009; Scott, 2007). In some networks, the high degree nodes are connected to high degree nodes. These are called assortative or homophilic networks. In disassortative networks high degree nodes avoid being connected to high degree nodes. These two types of networks are distinguished by using a degree-correlation coefficient that is positive for assortative networks and negative for disassortative networks (Newman, 2004). Assortative mixing seems more prevalent among social networks. However, both assortative and disassortative networks seem to occur in biological networks according to the requirement as to whether percolation or stability to fluctuations is needed.

The assortative networking results in larger positive Lyapunov exponents (eigenvalues) of the interacting matrix of the dynamical system. As the network size grows the real part of the eigenvalues scales as a power of the size of the network. This means the system can quickly become unstable resulting either in the formation of giant components in graph networks exhibiting the percolation phenomenon or collapsing like a house of cards. Such a phenomenon occurs in self-organising critical systems, such as ion channels. Here the sub units of proteins can alter their conformation to permit an ion to enter or exit through an interior hole connecting the inside of the cell and the exterior solution (Mosekilde and Mosekilde, 1991; Palla and Vattay, 2006).

In disassortative networking high degree nodes avoid being connected to high degree nodes and result in a smaller positive Lyapunov exponent (or positive eigenvalues); as the size of the network grows the real part of the eigenvalues have only a logarithmic dependence on the size; hence the dynamical fluctuations are not amplified and the system can reach stability more quickly. It has been observed that protein interactions happen in this manner (Murthy and Krishnamurthy, 2009).

In fact, according to Nowak's theorem (2006) the assortative networks work as amplifiers of selection, while disassortative networks tend to suppress selection.

\section{Motif-based graph growth for tuning GRNs}

Recent advances in cell biology reveals that nature has always concentrated on devising special purpose computing elements having both the analogue and digital features, and exploiting cooperative and competitive schemes using percolation like threshold phenomena, positive feedback leading to self-enforcement, non-linearity, and short and long range signalling to facilitate interaction using disordered protein networks. For accomplishing any special task, the protein machines are interconnected through softwiring, and are temporally and spatially coordinated through linked processes to 
achieve maximal efficiency. This problem is solved using genetic programming (GP) with motifs as the subgraphs in growing a graph.

Growing a graph until it reaches self-organised criticality through interaction is closely related to GP (Koza, 1999; Goldberg, 1989, 2002). In GP, each programme construct is a tree constructed from tasks, functions and terminal symbols. Then we perform crossover and mutation by swapping programme sub-trees leading to feasible programmes, taking care of the nature and type of the task. These operations resemble Metropolis-Hastings-Monte-Carlo methods to create transitivity in a graph from a given node to a desired attractor node. The GP operations correspond to an ergodic move-set in the space of graphs with a given set of parameters and repeatedly generating the moves and accepting them with probability $p$ or rejecting them with probability $(1-p)$.

Suitable move-sets are: creation of new nodes, aging and annihilation of nodes, mutation-movement of edges from one place to another, mating-swapping edges of the form $(s, t),(u, v)$ to $(s, u),(t, v)$, adding new edges based on a cost function. Such moves can create a phase transition (or percolation) to reach a global goal through successive local goals. An important aspect in GP is the fitness of the individual programme generated locally and globally. In self-organisation, ideally, one requires that the fitness is a self-awareness function, i.e., the individual who does the work evaluates itself, ensuring that the global fitness is guaranteed. This is widely prevalent in Nature for activities such as: nest building (stigmergy), food searching (foraging). For specified goals or for varying modularity this approach can be used to achieve special purpose genetic regulatory systems (Boccaletti et al., 2006). This approach is similar to using neural network methods.

As stated in Alon (2007a), the biological networks are modularly organised. In this sense, the conventional GP techniques as described above cannot capture the manner of biological evolution, since the random switching of edges, can destroy modularity. It is not easy retain the modular structure and vary the required connections to achieve a required goal.

Graph assembly models (Bornholdt and Schuster, 2003; Borrett et al., 2007) have also been used in the study of food webs. Here starting from a few species, new species are added by a process of invasion (assembly) or speciation (evolution) to generate large stable webs. This model is not realistic since the initial pool of species is predetermined, thereby limiting their choice and coevolved species cannot be taken into account. In food webs, it has been observed that there is modularity and these increase the stability. Most of these arguments are based on the eigenvalues of the adjacency matrices (Borrett et al., 2007).

Real systems including biological systems are non-stationary, in which the parameters are changing with time. Thus quantitative understanding of a $\mathrm{CN}$ using the above parameters with arbitrary time varying interactive topology is difficult. These difficulties arise due to the statistical nature of the models available and inability to compute the exact parameters numerically. However, for specific applications and a pre-defined static interactive topology among the motifs, the statistical parameters can be obtained through simulation and tuned to build a special purpose macro scale CN. Such a tuning scheme seems to have formed the basis behind anthropic coincidences and intelligent design. It is perhaps possible to use GP strategy to build such a $\mathrm{CN}$ for achieving selective goals. 
However, in using the motifs as building blocks for the genetic and evolutionary algorithm the fitness function can vary erratically due to inter and intra motif dependencies (local, inter-agent) and global dependency among these motifs (their relationship with other peripheral non-motifs) (Rothlauf, 2006; Sekanina, 2005; Goldberg, 2002). For specified goals or for varying modularity this approach can be used to achieve special purpose genetic regulatory systems (Alon, 2007a, 2007b). However, we are not able to establish this yet except for very simple circuits at the gate levels. It is hard to establish such fitness factors for functional and behavioural aspects of a Genetic regulatory system in a dynamic environment (Rothlauf, 2006).

Unlike in a deterministic algorithmic system, stochasticity or randomness and chaos seem to have provided a greater power to biological systems by creating an internal mechanism for exploration and discovery. As a result, in a biological system programmability is lost. The evolution of a resilient complex system is linked to its adaptability. Streamlining it through an algorithmic structure interferes with its self-organisation. Thus high adaptability is mutually exclusive to programmability; whenever maximum efficiency is needed adaptive systems can do better than programmable systems.

\section{Conclusions}

We examined the role of network motifs as a means to understand systems biology. Based on our arguments from a wide range of disciplines, it is difficult to conclude as to whether the approach based on an assembly of motifs can capture the functional properties of a composite GRN and can provide meaningful results in understanding life-like systems.

\section{References}

Alon, U. (2007a) An Introduction to System Biology, Chapman and Hall, New York.

Alon, U. (2007b) 'Network motifs: theory and experimental approaches', Nature Reviews (Genetics), June, Vol. 8, pp.450-461.

Boccaletti, S. et al. (2006) 'Complex networks: structure and dynamics', Physics Reports, January Vol. 424, pp.175-308.

Bornholdt, S. and Schuster, H.G. (Eds.) (2003) Handbook of Graphs and Networks, Wiley-VCH, Weinheim.

Borrett, S.R. et al. (2007) 'Functional integration of ecological networks through pathway proliferation', J. Theoretical Biology, Vol. 245, No. 1, pp.98-111.

Goldberg, D.E. (1989) Genetic Algorithms in Search, Optimisation and Machine Learning, Addison Wesley, Reading, Mass.

Goldberg, D.E. (2002) The Design of Innovation, Kluwer, Dordrecht.

Ingram, P.J. et al. (2006) 'Network motifs: structure does not determine function', BMC Genomics, May, Vol. 7, pp.108-121.

Jiang, R. et al. (2006) 'Network motif identification in stochastic networks', Proc. Natl. Acad. Sci., USA, Vol. 103, No. 25, pp.9404-9409.

Kashima, H., Tsuda, K. and Inokuchi, A. (2004) Kernel Methods in Computational Biology, in Scholopf, B., Tsuda, K. and Vert, J-P. (Eds.), pp.155-170, M.I.T. Press, Cambridge. 
Kitchen, L. and Krishnamurthy, E.V. (1982) 'Fast parallel relaxation screening for chemical patent database search', J. Chem. Inf. and Comput. Sci., Vol. 22, No. 1, pp.44-48.

Knabe, J.F., Nehaniv, C.L and Schilstra, M.I. (2007) 'Do motifs reflect evolved function? No convergent evolution of genetic regulatory network subgraph topologies', in 7th International Workshop on Information Processing in Cell and Tissues.

Koza, J.R. (1999) Genetic Programming III, Morgan Kaufmann, San Francisco.

Krishnamurthy, E.V. et al. (1974) 'ALWIN-algorithmic Wiswesser notation system for organic compounds', J. Chem. Doc., Vol. 14, No. 3, pp.130-141.

Krishnamurthy, E.V. and Lynch, M.F. (1981) 'Coding and analysis of generic chemical formulae in chemical patents', J. Inf. Sci., Vol. 3, No. 2, pp.75-79.

Manna, Z. (1974) Mathematical Theory of Computation, McGraw Hill, New York.

Mosekilde, E. and Mosekilde, L. (Eds.) (1991) Complexity, Chaos and Biological Evolution, Plenum Press, New York.

Murthy, V.K. and Krishnamurthy, E.V. (2009) 'Multiset of agents in a network for simulation of complex systems', in Kymakya, K. et al. (Eds.): Nonlinear Dynamics and Synchronization, Chapter 6, Springer, Berlin, 2010.

Newman, M.E.J. (2004) The Structure and Function of complex Networks, Santa Fe Institute, Birkhauser, Boston.

Nowak, M.A. (2006) Evolutionary Dynamics, The Belknap Press, Cambridge, Mass.

Palla, G. and Vattay, G. (2006) 'Spectral transitions in networks', New Journal of Physics, December, Vol. 8, pp.306-314.

Rothlauf, F. (2006) Representations for Genetic and Evolutionary Algorithms, Springer, New York.

Rupp, M. (2011) 'Graph kernels for chemoinformatics - a critical discussion', J. Cheminform., Vol. 3, Suppl. 1, p.O8.

Rupp, M. and Schneider, G. (2010) 'Graph kernels for molecular similarity', Molecular Informatics, Vol. 29, No. 5, pp.266-273.

Scott, A.C. (2007) The Nonlinear Universe, Springer, New York.

Sekanina, L. (2005) Evolvable Components, Springer, New York.

Shakhnovich, E. (2006) 'Protein folding thermodynamics and dynamics: where physics, chemistry and biology meet', Chemical Reviews, Vol. 106, No. 5, pp.1559-1588.

Villani, V. (2003) 'Complexity of polypeptide dynamics: chaos, Brownian motion and elasticity in aqueous solution', Journal of Molecular Structure: THEOCHEM, Vol. 621, No. 2, pp.127-139. 